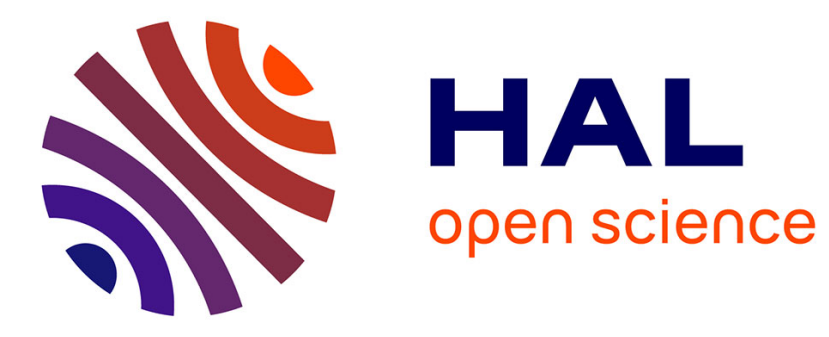

\title{
Les enjeux de l'apprentissage pour la personne polyhandicapée
}

\author{
Danièle Toubert-duffort
}

\section{To cite this version:}

Danièle Toubert-duffort. Les enjeux de l'apprentissage pour la personne polyhandicapée : Étude menée auprès de 18 élèves âgés de 4 à 14 ans, en contexte écologique de classe. La nouvelle revue - Éducation et société inclusives, 2020, 88, pp.65-85. 10.3917/nresi.088.0065 . hal-03507784

\section{HAL Id: hal-03507784 \\ https://hal-inshea.archives-ouvertes.fr/hal-03507784}

Submitted on 3 Jan 2022

HAL is a multi-disciplinary open access archive for the deposit and dissemination of scientific research documents, whether they are published or not. The documents may come from teaching and research institutions in France or abroad, or from public or private research centers.
L'archive ouverte pluridisciplinaire HAL, est destinée au dépôt et à la diffusion de documents scientifiques de niveau recherche, publiés ou non, émanant des établissements d'enseignement et de recherche français ou étrangers, des laboratoires publics ou privés. 


\title{
Les enjeux de l'apprentissage pour la personne polyhandicapée
}

\author{
Étude menée auprès de 18 élèves \\ âgés de 4 à 14 ans, \\ en contexte écologique de classe
}

\author{
Danièle TOUBERT-DUFFORT \\ Maître de conférences en psychologie \\ Psychologue clinicienne \\ Grhapes, EA 7287, INSHEA
}

Résumé: $\quad$ Depuis la loi n² 2005-102 du 11 février 2005, le droit des jeunes polyhandicapés à suivre un enseignement est affirmé, ce qui implique pour l'école de se rendre accessible à ces jeunes malgré l'ampleur de leurs difficultés cognitives. À partir d'une étude inductive menée dans cinq unités d'enseignement et auprès de 18 jeunes, âgés de 4 à 14 ans, nous cherchons à préciser quelles sont les modalités d'apprentissage des jeunes en situation de polyhandicap. Nous montrons qu'il existe une diversité des configurations cliniques. Les apprentissages sont effectifs mais dépendent étroitement du contexte pédagogique et relationnel. L'enseignant remplit une fonction d'étayage et de médiation sans laquelle il n'y a pas d'interrelation fonctionnelle.

Mots-clés: Accessibilité pédagogique-Personne polyhandicapée-Processus d'apprentissage-Recherche participative.

The challenges of learning for people with Profound Intellectual Multiple Disabilities (PIMD) Study conducted with 18 pupils aged 4 to 14, in an ecological classroom context

Summary: Since the law $n^{\circ} 2005-102$ of February 11 th, 2005, the right of the people with Profound Intellectual Multiple Disabilities (PIMD) to be a pupil is affirmed, which implies for the school to be made accessible to these young people in spite of the extent of their cognitive disabilities. Based on a study conducted in five teaching units with 18 young people, aged 4 to 14, this article examines learning situations in a class ecological context, in order to specify the processes and issues involved learning for this people. We show that there is a diversity of clinical configurations. Learning is effective but depends closely on the pedagogical and relational context. The teacher performs a supporting and mediation function without which there is no functional relationship.

Keywords: Learning process (for people with PIMD) - Participatory research - People with Profound Intellectual Multiple Disabilities (PIMD) - Special education. 
Depuis l'affirmation du droit à la scolarité pour tout enfant (cf. loi 2005-102 du 11 février 2005) - y compris avec polyhandicap - réaffirmé par la loi 2013-595 du 8 juillet 2013 qui dispose que le " service public de l'éducation [...] veille à l'inclusion scolaire de tous les enfants, sans aucune distinction " (article 111-1), et la création des unités d'enseignement (2009), c'est en étroite collaboration avec l'Éducation nationale que les établissements et services médico-sociaux ont pour mission de répondre aux besoins d'apprentissage des jeunes polyhandicapés, alors même que l'école doit se rendre accessible à tous.

Le polyhandicap caractérise " une situation de vie spécifique d'une personne présentant un dysfonctionnement cérébral, précoce ou survenu en cours de développement, ayant pour conséquences de graves perturbations à expressions multiples et évolutives de l'efficience motrice, perceptive, cognitive et de la construction des relations avec l'environnement physique et humain " (GPF, 2002). Des " handicaps neurologiques précoces " qui peuvent, selon Ouss (2019), entraîner une situation lourde d'implications, à la fois pour l'ensemble des processus développementaux de la personne et pour la construction psychique du sujet.

En France, selon la dernière enquête de la Drees publiée en juillet 2018 sur la situation au 31 décembre 2014, l'accès aux apprentissages scolaires en contexte d'inclusion n'est envisagé que rarement, pour 1,5\% des jeunes en situation de polyhandicap, et il reste minoritaire même dans le cadre privilégié de l'unité d'enseignement interne à l'établissement spécialisé ( $21 \%$ des jeunes, selon la même enquête). Ce faible taux de scolarisation est lié à la complexité du polyhandicap (Saulus, 2007), aux besoins très spécifiques de la personne polyhandicapée, encore méconnus, en particulier sur le plan de l'accessibilité pédagogique, et à la relative non expertise des professionnels de l'éducation (Camberlein, 2011). Par ailleurs, nous disposons encore de peu de connaissances sur les compétences cognitives et socio-émotionnelles de la personne polyhandicapée, et en conséquence d'outils fiables pour les évaluer dans toute leur diversité et leur atypicité (Chard et Roulin, 2015). Enfin, il n'est pas rare que l'on dénie à la personne polyhandicapée l'existence même d'une subjectivité, tant l'accès à celle-ci, faute de langage, est rendu complexe (Toubert, 2011). L'objectif de cet article est d'analyser les enjeux de l'apprentissage en contexte scolaire, s'agissant d'enfant ou d'adolescent en situation de polyhandicap, à partir d'une étude menée auprès de 18 jeunes, âgés de 4 à 14 ans. Dans une première partie, nous examinerons les fondements théoriques qui ont guidé notre approche conceptuelle de l'apprenant, en situation de polyhandicap. Nous présenterons ensuite le dispositif méthodologique, puis nous discuterons des processus mobilisés chez l'apprenant, et des enjeux de la scolarisation.

\section{CONTEXTUALISATION DE LA PROBLÉMATIOUUE ET ANCRAGE CONCEPTUEL}

\section{Une dynamique intersubjective à construire, un positionnement éthique qui ne va pas de soi}

Dans la situation de polyhandicap, les aspects corporels et médicaux, prédominants et prégnants, produisent une certaine distorsion du regard jusqu'à provoquer une forme 


\section{DOSSIER}

de sidération qui entrave les capacités d'entrée en relation de celui qui accompagne, soigne, éduque ou enseigne la personne polyhandicapée. La rencontre avec cet autre à la fois si semblable et si étrangement différent constitue bien souvent une énigme, produit des images ou des vécus angoissants (Korff-Sausse, 2008), et font courir à la personne polyhandicapée le risque d'être confrontée à une perception négative (Blondel et Delzescaux, 2018). Entre reconnaissance et déni de réalité, la posture des aidants professionnels ou familiaux est le plus souvent marquée par l'ambivalence dans les relations à l'égard de la personne en situation de polyhandicap (Blondel et Delzescaux, 2018).

Le polyhandicap requiert d'abord du professionnel qu'il (re)pense sa posture: d'une part en veillant à affiner ses capacités d'observation et d'attention, afin de pouvoir faire la distinction entre ce qui peut être support de progrès pour une personne, et ce qui peut lui faire violence, la frontière étant parfois ténue (Toubert-Duffort, 2011) ; d'autre part, en développant une écoute exclusivement centrée sur la personne, sans préoccupation narcissique quant à sa propre personne ou sa professionnalité (Saulus, 2007). Les enjeux de notre posture de chercheur rejoignent en partie ceux des professionnels et des parents impliqués dans l'accompagnement pédagogique de la personne polyhandicapée: comment apprécier l'enfant en situation de polyhandicap sous l'angle de ses compétences et de ses aptitudes? Comment accéder à ses manières singulières d'être au monde, si discrètes soient-elles? Comment se rendre suffisamment sensible à des processus si difficiles à percevoir et à interpréter?

Le polyhandicap, sous l'angle des apprentissages et de la scolarisation, nous conduit ensuite à interroger plus spécifiquement les itinéraires d'apprentissage de la personne polyhandicapée, de manière à concevoir un environnement et un accompagnement pédagogique qui soient le plus adapté possible au développement de l'élève en situation de polyhandicap.

\section{D'une conception interactionniste du développement, aux différents modèles permettant d'appréhender les enjeux de l'apprentissage pour la personne polyhandicapée}

Aujourd'hui prévaut une conception interactionniste du développement, en référence au modèle biopsychosocial de l'individu (Engel, 1977): on considère que le développement résulte de l'action conjointe des facteurs biologiques internes et des variables de l'environnement, en particulier celles du monde social. Cette perspective, dans laquelle nous nous situons, implique à l'égard de notre objet d'étude, l'élève avec polyhandicap, en contexte d'apprentissage, la prise en compte de l'interaction entre des facteurs individuels d'une part (l'enfant, ses déficiences, son profil, son histoire...) et des facteurs environnementaux d'autre part (les professionnels, les parents, les pairs...).

Trois approches sont actuellement privilégiées pour aborder la problématique de la personne polyhandicapée en tant qu'apprenant, et sujet en développement: une approche neuro-développementale et cognitive qui s'appuie sur les travaux renseignant les formes et les processus que prend la cognition naissante; une approche clinique et phénoménologique qui s'intéresse spécifiquement à la structure du polyhandicap et à l'activité psychique (en particulier le développement de la conscience de soi et 
des processus de représentation); et une approche psycho-éducative, peu développée en France, centrée sur le contexte d'apprentissage (interactions entre pairs...) et les pratiques professionnelles.

Nous savons que certains facteurs favorisent la maîtrise instrumentale comme par exemple l'encodage multiple d'une même propriété qui aide à l'intégration de celle-ci (Auvray, 2005). Les premières activités instrumentales émergent ainsi progressivement d'un fonctionnement biologique " pré-câblé ", à condition que le bébé puisse accéder à des régularités, des routines permettant l'extraction d'invariants (Bullinger, 2007). Ainsi en est-il par exemple de l'activité de succion, qui, à partir d'un certain moment, ne répondra plus uniquement au service des fonctions prédéterminées, telles que la succion, l'apaisement ou l'exploration, mais pourra en être détournée et devenir une activité instrumentale intelligente (Bruner, 1968). Dans cette perspective cognitive et neuro-développementale, deux principes accompagnent les premières étapes du développement et de l'apprentissage:

- d'une part, l'activité cognitive est en étroite relation avec les autres fonctions, perceptives, motrices et émotionnelles. Pour les tenants du paradigme de la " cognition incarnée " (Suchman, 1987), elle ne saurait être dissociée de l'engagement corporel et moteur, la cognition prenant racine dans le corps, dans ses composantes émotionnelles et sensori-motrices les plus profondes. Cette théorie nous permet de mieux saisir en quoi les multiples déficiences dont sont atteintes les personnes polyhandicapées s'entretiennent mutuellement, ajoutant du handicap au handicap, dans une boucle dynamique et permanente (Chard et Roulin, 2015). Par ailleurs, elle permet d'envisager le corps de la personne polyhandicapée, à travers ses dimensions aussi bien motrices qu'émotionnelles, comme fondement même de toute activité cognitive. Cette activité cognitive peut naître, croître et/ou se maintenir grâce aux interactions entre l'individu et son environnement, à travers les objets qu'il rencontre et les situations d'apprentissage dans lesquelles il est engagé.

- D'autre part, la conception Vygotskienne du développement du psychisme enfantin insiste sur la fonction d'étayage et de médiation assurée par les adultes, mettant en avant les outils de communication, en particulier le langage, comme vecteur principal d'apprentissage (Vygotski, 1934). L'enfant élaborerait ses connaissances lors des échanges avec de plus experts que lui (en général les adultes ayant mission de l'instruire), à condition que ceux-ci interviennent dans la "zone de proche développement ": ce qui fonctionne d'abord dans I'interrelation est progressivement intériorisé. Au départ, l'adulte oriente l'activité de l'enfant, la soutient et la contrôle; les différentes fonctions auquel I'enfant, étayé par l'adulte, accède, deviennent progressivement auto-régulées, en l'absence de trouble. L'importance de la fonction d'étayage de l'éducateur, reprise et développée par Bruner, a donné lieu à tout un courant de travaux relatifs aux interactions mère-enfant, essentiellement aux premiers âges de la vie, au moment où se construisent les compétences cognitives à la base des apprentissages plus complexes: discrimination, catégorisation, attention partagée, attention conjointe, théorie de l'esprit, apprentissage du langage, etc. (Weil-Barais et Resta-Schweitzer, 2008). 


\section{DOSSIER}

S'agissant de l'activité mentale de la personne polyhandicapée, elle se caractérise par une forme de représentation tout à fait particulière fonctionnant à partir de matériaux sensoriels et moteurs en dehors de l'apparition du langage: les proto- ou pré-représentations. Ces " proto-représentations correspondent à une forme d'activité mentale étayée par l'action, qui faute de dimension symbolique, s'actualise uniquement dans une situation en temps réel et non pas hors situation en temps différé »(Petitpierre-Jost, 2005, p. 39). En référence à une approche clinique, Saulus $(2008,2009,2017)$ propose un modèle structural du polyhandicap, avec une description du développement de l'activité mentale de la personne polyhandicapée en trois profils: ces profils psycho-développementaux permettent de rendre compte de l'évolution d'un éprouvé archaïque d'existence (profil 1) vers un éprouvé entitaire d'existence (profil 3), progressivement, au fil du développement sensori-moteur, cognitif et affectif. Les travaux de Juliane Dind (2018) valident empiriquement l'existence de ces profils, en continuité les uns avec les autres. Le modèle décrit par Saulus s'étaye largement sur une conception phénoménologique qui fait de l'éprouvé une activité psychique (ou protopsychique lorsqu'il s'agit des toutes premières étapes du développement) fondamentale. Cette activité donne lieu à ce qu'on nomme des éprouvés, qui mêlent cognition et affectivité. Le développement des processus archaïques et primaires (constitution du sentiment continu d'existence, proto-représentations, premières expériences du Moi, premières représentations, premières distinctions d'avec l'objet et d'avec autrui) n'est rendu possible que dans le lien à l'environnement humain. C'est en effet celui-ci qui va créer les conditions requises pour permettre l'émergence, puis la stabilisation, d'un éprouvé et d'une capacité représentative, soubassement du sentiment continu d'existence et de l'émergence du Moi.

Plus directement centrée sur l'apprentissage et l'analyse des interactions entre la personne polyhandicapée et ses partenaires, l'approche psycho-éducative met en évidence l'intérêt de situations d'apprentissage mobilisant des acquis relevant simultanément des domaines cognitif, communicationnel, psychomoteur et socioémotionnel (Hostyn, Maes et Neerinckx, 2009, 2011). La capacité à prendre conscience des stratégies d'interrelation avec la personne polyhandicapée, le contexte dans lequel celles-ci se déroulent, ainsi que la perception que l'on a de ces personnes sont autant de variables qui interviennent dans les potentialités d'expression de leurs compétences et dans les apprentissages (Neerinckx et Maes, 2016). Par ailleurs le contexte de polyhandicap fait aussi obstacle aux échanges entre pairs (Nijs, Vlaskamp et Maes, 2015).

La communauté scientifique s'accorde sur la nécessité d'une approche à la fois plurielle, discutant le polyhandicap tant du point de vue de sa spécificité (neurophysiologique et neuropsychopathologique) que du retentissement des troubles sur la construction du sujet, et multifactorielle, se situant hors d'une logique de causalité linéaire (Ouss, 2019). Cependant, peu de travaux explorent les processus psychiques mobilisés par la personne polyhandicapée en contexte écologique d'apprentissage, la plupart des recherches s'appuyant sur des dispositifs expérimentaux.

Inscrivant notre travail dans le champ de la clinique de l'éducation, nous nous intéressons à l'élève en situation de polyhandicap, en tant que sujet apprenant 
singulier (avec son histoire et sa subjectivité propre, aussi difficile d'accès soitelle), et à ce qui se joue pour lui en contexte d'apprentissages scolaires. Dans cette perspective, c'est dans l'interrelation avec son environnement humain que le sujet peut naître à lui-même, contre ou malgré le contexte dé-subjectivant du polyhandicap. Nous considérons, au regard des différents travaux recensés qu'il ne suffit pas de construire un cadre, de solliciter les actions et les interactions pour que l'élève en situation de polyhandicap puisse en tirer profit sur le plan de ses apprentissages, et que s'instaure une interrelation fonctionnelle. Les objectifs de notre recherche sont d'appréhender les modalités d'apprentissage des jeunes en situation de polyhandicap, à partir d'une analyse individuelle de leur profil d'une part, et d'une analyse de leurs comportements en contexte de classe d'autre part. Nous tenterons ainsi de répondre aux questions suivantes: que nous apprend l'évaluation individuelle et fonctionnelle de ces jeunes sur leurs potentialités d'apprentissage? Que nous apprend leur observation en contexte écologique de classe, s'agissant de leurs manières singulières d'être au monde, d'agir ou de réagir face à leur environnement et aux sollicitations des adultes engagés vis-à-vis d'eux dans une tâche d'enseignement et d'éducation?

\section{MÉTHODOLOGIE}

Compte tenu de l'absence de références spécifiques à notre objet d'étude et du caractère exploratoire de notre recherche, nous avons privilégié une démarche inductive et qualitative: nous sommes partis des faits observés (évaluation individuelle des profils cognitifs, évaluation des comportements en contexte de classe) pour construire du sens, tenter de dégager des invariants, et répondre à nos objectifs de recherche. L'analyse inductive nous a permis de:

- condenser des données brutes de différentes natures (données historiques et personnelles sur l'enfant, recueillies à partir d'entretiens avec les parents; données issues de l'évaluation psychologique; données issues des séquences pédagogiques filmées) dans un format résumé, de type étude de cas, pour chaque jeune;

- établir des liens entre les objectifs de la recherche et les différentes catégories découlant des données brutes réduites (études de cas). À partir d'une analyse comparative et transversale de l'ensemble de ces données, nous avons cherché à dégager les composantes cliniques des situations de polyhandicap, à en préciser le caractère hétérogène et à interroger les processus psychiques mobilisés par les jeunes lors des séances pédagogiques.

Des précautions méthodologiques ont été prises, pour limiter le caractère subjectif de la démarche: nous avons procédé à un double codage pour l'analyse des séquences pédagogiques filmées, et pris soin de lire à plusieurs reprises les données brutes. Nous avons par ailleurs privilégié une évaluation dynamique, multidimensionnelle et fonctionnelle:

- Une évaluation dynamique: nous avons analysé, pour chacun des jeunes, trois séances pédagogiques, une par trimestre. De même nous avons procédé à deux évaluations par le P2CJP, en début et en fin de recherche, 18 mois plus tard. 


\section{DOSSIER}

- Une évaluation multidimensionnelle et interdisciplinaire: les observations des professionnels (enseignant, psychomotricien, kinésithérapeute, psychologue, AMP) ont été croisées entre elles et avec celles des parents.

- Une évaluation fonctionnelle: elle s'est faite sur la base d'activités signifiantes pour les jeunes, proposées dans le cadre écologique de la classe par les enseignants qui les connaissaient bien. Les comportements ont été analysés en relation avec les facteurs contextuels, relationnels et les objectifs visés.

\section{Choix paradigmatique d'une approche participative}

L'observation longitudinale des jeunes en situation de polyhandicap a constitué le cœur de la procédure autour duquel le reste de la démarche de recherche a été construite. L'observation des jeunes en situation de polyhandicap est fondamentale dans la mesure où elle se réfère à une démarche compréhensive qui interroge à la fois la dimension objective des comportements observés et la dimension relationnelle en jeu dans l'intersubjectivité du lien. En effet, puisque nous ne pouvons (le plus souvent) utiliser le langage ou un mode de communication symbolique pour appréhender avec certitude ce que ces jeunes comprennent, ressentent et apprennent, notre principal mode d'accès à leurs compétences a été l'observation. Les difficultés d'appréciation des compétences cognitives de ces personnes font courir des risques connus, à savoir surestimer ou au contraire sous-estimer leurs aptitudes et compétences (Pereira Da Costa et Scelles, 2012). Ainsi, les proches, parents et référents du jeune ont été des informateurs incontournables pour nous aider dans l'évaluation des processus mobilisés par les jeunes en situation écologique d'apprentissage. Notre démarche, clinique, a privilégié:

- une première phase de mobilisation et d'implication des acteurs concernés (parents et professionnels) autour de l'évaluation de l'enfant;

- une observation continue, qui s'est opérée dans des contextes relationnels et situationnels différents et qui a impliqué différents acteurs (parent, enseignant(s), professionnel du soin, éducateur, AMP, AVS) aux côtés des chercheurs ;

- un retour réflexif sur les observations menées, dans le cadre de petits groupes de discussion pour croiser les regards.

Notre méthodologie d'observation a associé une observation structurée par des grilles (P2CJP, grille d'observation des séances pédagogiques filmées), et une observation relationnelle (réunions d'analyse en regards croisés) dont la visée a été d'éclairer les dimensions subjectives et intersubjectives en jeu dans les interactions.

\section{Population}

Les 18 jeunes de l'étude sont accueillis dans cinq établissements spécialisés qui possèdent chacun une unité d'enseignement.

- Ils sont âgés de 4 à 14 ans.

- Ils sont en situation de polyhandicap, selon la définition du GPF.

- Il est impossible de définir un niveau intellectuel par des tests standardisés (cf. critère PIMD).

- Ils ont peu ou pas de compréhension du langage verbal, pas d'accès aux interactions d'ordre symbolique, aucune capacité à se gérer par eux-mêmes (cf. critère PIMD) ; 
en sus des déficits sensoriels, ils présentent un risque élevé de complications sur le plan de leur santé, ce qui en fait des personnes vulnérables (cf. critère PIMD). - Ils bénéficient, dans le cadre du projet de recherche, de deux temps de scolarisation par semaine.

En référence au modèle décrit par Ponsot et Boutin (2017), nous avons classé en trois catégories les étiologies recensées dans notre étude. II en ressort que:

83,3 \% des jeunes de l'étude relèvent d'un polyhandicap congénital fixé dont les causes sont liées pour 73,3 \% d'entre eux à des problèmes d'ordre génétique, malformatif, infectieux ou vasculaire et $26,7 \%$ sont d'origine inconnue. $11 \%$ des jeunes relèvent d'un polyhandicap congénital évolutif. 5,6 \% des jeunes relèvent d'un polyhandicap acquis d'origine infectieuse, vasculaire ou accidentelle. Nous retrouvons un pourcentage similaire à l'étude citée par Ponsot et Boutin (2017) concernant le polyhandicap congénital fixé qui constitue la première cause répertoriée du polyhandicap.

\section{SYNTHĖSE DES RÉSULTATS ET DISCUSSION}

\section{Que nous apporte l'évaluation individuelle, multidimensionnelle et interdisciplinaire des jeunes en situation de polyhandicap, en amont de la scolarisation?}

Cette évaluation initiale s'est faite sur la base d'un entretien conduit par le (la) psychologue en présence des référents médicaux et éducatifs de l'enfant et de ses parents, autour d'observations partagées, de façon à établir le profil du jeune. Pour ce faire, nous avons interrogé les situations, à la fois au regard des limitations imposées par le contexte de polyhandicap (tableau 1), mais aussi au regard des compétences cognitives sous-jacentes aux apprentissages, en particulier l'attention, la mémoire et la communication. 
Tableau 1 : Description des situations de polyhandicap

\begin{tabular}{|c|c|c|c|c|c|}
\hline Noms & $\begin{array}{l}\text { Type de } \\
\text { polyhandicap }\end{array}$ & $\begin{array}{l}\text { Déficience intellectuelle sévère } \\
\text { et Déficience motrice }\end{array}$ & $\begin{array}{l}\text { Troubles } \\
\text { psychiques }\end{array}$ & $\begin{array}{l}\text { Déficiences } \\
\text { neurosensorielles }\end{array}$ & Troubles somatiques \\
\hline Maxence & $\begin{array}{l}\text { Polyhandicap } \\
\text { fixé congénital } \\
\text { génétique }\end{array}$ & Hypertonie & $\begin{array}{l}\text { Troubles psycho- } \\
\text { émotionnels } \\
\text { Stéréotypies } \\
\text { Comportements } \\
\text { automutilation }\end{array}$ & $\begin{array}{l}\text { Hypersensibilité } \\
\text { tactile }\end{array}$ & $\begin{array}{l}\text { Troubles alimentaires (nourri } \\
\text { par sonde gastrostomique) }\end{array}$ \\
\hline Max & $\begin{array}{l}\text { Polyhandicap } \\
\text { fixé congénital } \\
\text { génétique }\end{array}$ & $\begin{array}{l}\text { Quadriplégie } \\
\text { Troubles du tonus global }\end{array}$ & $\begin{array}{l}\text { Troubles psycho- } \\
\text { émotionnels }\end{array}$ & & Épilepsie stabilisée \\
\hline Janine & $\begin{array}{l}\text { Polyhandicap } \\
\text { acquis d'origine } \\
\text { accidentelle ou } \\
\text { infectieuse }\end{array}$ & Quadriplégie & $\begin{array}{l}\text { Comportements } \\
\text { automutilation }\end{array}$ & & $\begin{array}{l}\text { Épilepsie partiellement } \\
\text { stabilisée }\end{array}$ \\
\hline Inès & $\begin{array}{l}\text { Polyhandicap } \\
\text { congénital } \\
\text { évolutif }\end{array}$ & $\begin{array}{l}\text { Troubles des membres inférieurs } \\
\text { Trouble du tonus global } \\
\text { (spasticité) }\end{array}$ & $\begin{array}{l}\text { Troubles du } \\
\text { spectre autistique }\end{array}$ & $\begin{array}{l}\text { Hypersensibilité } \\
\text { aux stimulations } \\
\text { extérieures (bruits, } \\
\text { lumière) qui peuvent } \\
\text { provoquer crises } \\
\text { épilepsie }\end{array}$ & $\begin{array}{l}\text { Épilepsie partiellement } \\
\text { stabilisée }\end{array}$ \\
\hline Driss & $\begin{array}{l}\text { Polyhandicap } \\
\text { fixé congénital } \\
\text { malformatif }\end{array}$ & Quadriplégie & $\begin{array}{l}\text { Troubles psycho- } \\
\text { émotionnels } \\
\text { Comportements } \\
\text { perturbateurs }\end{array}$ & & Épilepsie stabilisée \\
\hline Colin & $\begin{array}{l}\text { Polyhandicap } \\
\text { fixé congénital } \\
\text { génétique }\end{array}$ & Troubles des membres inférieurs & Stéréotypies & $\begin{array}{l}\text { Trouble visuel } \\
\text { (champ rétréci) }\end{array}$ & Troubles respiratoires \\
\hline
\end{tabular}




\begin{tabular}{|c|c|c|c|c|c|}
\hline Noms & $\begin{array}{l}\text { Type de } \\
\text { polyhandicap }\end{array}$ & $\begin{array}{l}\text { Déficience intellectuelle sévère } \\
\text { et Déficience motrice }\end{array}$ & $\begin{array}{l}\text { Troubles } \\
\text { psychiques }\end{array}$ & $\begin{array}{l}\text { Déficiences } \\
\text { neurosensorielles }\end{array}$ & Troubles somatiques \\
\hline Jules & $\begin{array}{l}\text { Polyhandicap } \\
\text { fixé congénital } \\
\text { inconnu }\end{array}$ & $\begin{array}{l}\text { Quadriplégie } \\
\text { Troubles du tonus global } \\
\text { (hypotonique) }\end{array}$ & Auto-agression & $\begin{array}{l}\text { Myopie } \\
\text { Surdité profonde }\end{array}$ & $\begin{array}{l}\text { Troubles de la déglutition } \\
\text { Troubles du sommeil }\end{array}$ \\
\hline Jeanne & $\begin{array}{l}\text { Polyhandicap } \\
\text { fixé congénital } \\
\text { malformatif }\end{array}$ & $\begin{array}{l}\text { Quadriplégie } \\
\text { Hypertonie }\end{array}$ & $\begin{array}{l}\text { Troubles psycho- } \\
\text { émotionnels }\end{array}$ & $\begin{array}{l}\text { Cécité partielle, } \\
\text { difficile à évaluer } \\
\text { Hypersensibilité } \\
\text { tactile }\end{array}$ & Troubles digestifs \\
\hline Agathe & $\begin{array}{l}\text { Polyhandicap } \\
\text { fixé congénital } \\
\text { inconnu }\end{array}$ & $\begin{array}{l}\text { Troubles des membres inférieurs } \\
\text { (main droite) }\end{array}$ & & $\begin{array}{l}\text { Trouble visuel } \\
\text { (strabisme } \\
\text { convergent) }\end{array}$ & $\begin{array}{l}\text { Troubles du sommeil } \\
\text { Épilepsie stabilisée }\end{array}$ \\
\hline Éléonore & $\begin{array}{l}\text { Polyhandicap } \\
\text { fixé congénital } \\
\text { génétique }\end{array}$ & $\begin{array}{l}\text { Troubles du tonus global } \\
\text { Troubles de l'équilibre }\end{array}$ & $\begin{array}{l}\text { Troubles psycho- } \\
\text { émotionnels } \\
\text { Comportements } \\
\text { perturbateurs }\end{array}$ & & Troubles du sommeil \\
\hline Cyrielle & $\begin{array}{l}\text { Polyhandicap } \\
\text { fixé congénital } \\
\text { inconnu }\end{array}$ & $\begin{array}{l}\text { Troubles moteurs des } 4 \\
\text { membres } \\
\text { Trouble du tonus global }\end{array}$ & & Surdité légère & \\
\hline Mona & $\begin{array}{l}\text { Polyhandicap } \\
\text { fixé congénital } \\
\text { génétique }\end{array}$ & Hypotonie des membres & $\begin{array}{l}\text { Autostimulation, } \\
\text { stéréotypies }\end{array}$ & Cécité & \\
\hline Nolan & $\begin{array}{l}\text { Polyhandicap } \\
\text { fixé congénital } \\
\text { inconnu }\end{array}$ & $\begin{array}{l}\text { Quadriplégie } \\
\text { Troubles du tonus global }\end{array}$ & $\begin{array}{l}\text { Troubles du } \\
\text { comportement } \\
\text { (agrippement) }\end{array}$ & Trouble auditif & \\
\hline
\end{tabular}




\begin{tabular}{|c|c|c|c|c|c|}
\hline Noms & $\begin{array}{l}\text { Type de } \\
\text { polyhandicap }\end{array}$ & $\begin{array}{l}\text { Déficience intellectuelle sévère } \\
\text { et Déficience motrice }\end{array}$ & $\begin{array}{l}\text { Troubles } \\
\text { psychiques }\end{array}$ & $\begin{array}{l}\text { Déficiences } \\
\text { neurosensorielles }\end{array}$ & Troubles somatiques \\
\hline Abdel & $\begin{array}{l}\text { Polyhandicap } \\
\text { fixé congénital } \\
\text { malformatif }\end{array}$ & $\begin{array}{l}\text { Hypotonie généralisée et } \\
\text { tétraplégie }\end{array}$ & $\begin{array}{l}\text { Autostimulation, } \\
\text { stéréotypies } \\
\text { (mains dans la } \\
\text { bouche) } \\
\text { Auto-agression } \\
\text { (mord sa main) }\end{array}$ & $\begin{array}{l}\text { Hypersensibilité } \\
\text { visuelle et tactile }\end{array}$ & $\begin{array}{l}\text { Épilepsie partiellement } \\
\text { stabilisée }\end{array}$ \\
\hline Faustine & $\begin{array}{l}\text { Polyhandicap } \\
\text { fixé congénital } \\
\text { malformatif }\end{array}$ & $\begin{array}{l}\text { Quadriplégie } \\
\text { Trouble du tonus global, agitation }\end{array}$ & $\begin{array}{l}\text { Troubles psycho- } \\
\text { émotionnels } \\
\text { Stéréotypies } \\
\text { Parfois } \\
\text { automutilation } \\
\text { (se mord) }\end{array}$ & & $\begin{array}{l}\text { Épilepsie stabilisée } \\
\text { Problèmes ORL }\end{array}$ \\
\hline Adam & $\begin{array}{l}\text { Polyhandicap } \\
\text { fixé congénital } \\
\text { inconnu }\end{array}$ & $\begin{array}{l}\text { Quadriplégie } \\
\text { Trouble du tonus global }\end{array}$ & $\begin{array}{l}\text { Trouble du } \\
\text { spectre autistique }\end{array}$ & $\begin{array}{l}\text { Surdité difficile à } \\
\text { évaluer } \\
\text { Trouble visuel (port } \\
\text { de lunettes) } \\
\text { Hypersensibilité } \\
\text { (visage) }\end{array}$ & Épilepsie non stabilisée \\
\hline Talia & $\begin{array}{l}\text { Polyhandicap } \\
\text { évolutif } \\
\text { congénital }\end{array}$ & $\begin{array}{l}\text { Trouble du tonus général: } \\
\text { raideurs, difficulté à tenir sa tête } \\
\text { Quadriplégie }\end{array}$ & $\begin{array}{l}\text { Troubles psycho- } \\
\text { émotionnels } \\
\text { Stéréotypies }\end{array}$ & $\begin{array}{l}\text { Trouble visuel (port } \\
\text { de lunettes) }\end{array}$ & Troubles de la déglutition \\
\hline Clotilde & $\begin{array}{l}\text { Polyhandicap } \\
\text { fixé congénital } \\
\text { neuro-génétique }\end{array}$ & $\begin{array}{l}\text { Trouble du tonus généralisé } \\
\text { (agitation) } \\
\text { Quadriplégie }\end{array}$ & $\begin{array}{l}\text { Hyperactivité } \\
\text { Troubles psycho- } \\
\text { émotionnels } \\
\text { Stéréotypies } \\
\text { Auto-agression }\end{array}$ & Trouble visuel & $\begin{array}{l}\text { Épilepsie stabilisée } \\
\text { Troubles du sommeil }\end{array}$ \\
\hline
\end{tabular}




\section{Un accès aux apprentissages complexifié par des limitations importantes}

On retrouve dans notre population les traits spécifiques au polyhandicap constitué par l'intrication de deux déficiences principales: une déficience mentale sévère à profonde associée à une déficience motrice grave. Ces déficiences principales sont également presque toujours associées à d'autres troubles, psychiques, neurosensoriels et somatiques (Boutin et Ponsot, 2017). L'analyse de notre échantillon montre toutefois que ces troubles ne sont pas tous présents chez chacun des jeunes, qu'ils ne se présentent pas sous les mêmes modalités, et qu'ils peuvent être intriqués. Dans le contexte du polyhandicap, les altérations subies par l'équipement neurologique pour des raisons génétiques, malformatives, infectieuses, traumatiques ou toxiques impactent massivement la maturation et l'apprentissage spontané. Ces résultats confirment l'hypothèse selon laquelle la tâche de l'environnement social se trouve accrue, l'accès aux apprentissages étant largement complexifié par les limitations liées au polyhandicap. L'enseignant, comme les autres professionnels, va donc être conduit à déjouer, dans la mesure du possible, les répercussions dues aux troubles neurologiques, de façon à prévenir la survenue de surhandicaps, en particulier l'aggravation du déficit cognitif par l'accumulation de déficiences multiples et l'absence de compensations. Ces surhandicaps affectent la communication, l'activité ou la participation, et influent sur la qualité de vie. La situation de l'enfant polyhandicapé peut ainsi être aggravée ou bien améliorée par l'environnement, d'où la nécessité d'évaluer très régulièrement si l'environnement apporte des éléments facilitant ou au contraire limitant l'activité et la participation, affectant la qualité de vie de la personne polyhandicapée.

\section{Des potentialités d'apprentissage}

\section{avec une hétérogénéité intra et interindividuelle des profils cognitifs}

Des compétences attentionnelles et mnésiques sont présentes chez tous les jeunes mais à des degrés divers. Fluctuantes et labiles, elles dépendent de nombreux facteurs propres à chacun: ses états d'éveil, sa motivation, son état de santé (sédation de la douleur, crises d'épilepsie, absences, fatigabilité...), la nature et l'ampleur de ses déficits perceptifs, son sentiment de sécurité interne, et enfin sa faible capacité à mettre à l'écart l'information non pertinente (processus d'inhibition). Les compétences communicationnelles reposent majoritairement sur des modalités non verbales, plus riches et nombreuses que les modalités langagières, en émission comme en réception.

Le tableau ci-dessous présente une analyse synthétique des modalités de communication que nous avons identifiées chez les jeunes de notre étude, à partir des évaluations initiales par le P2CJP, en amont de la scolarisation. 


\section{DOSSIER}

Tableau 2: Communication

Communication orale du jeune

Réception

- Comprend quelques mots

- Comprend des phrases courtes

Émission

- Émet des sons difficiles à interpréter

- Émet des sons généralement interprétables

- Énonce quelques mots

\section{Communication non verbale}

\section{Réception}

- Perçoit et/ou comprend les émotions d'autrui

- Montre qu'il/elle comprend qu'on s'adresse à lui/elle par sa gestuelle et ses expressions faciales

\section{Émission}

- Pas d'intention de communiquer décelable

- Exprime différentes émotions (colère, tristesse, joie)

- Réagit à un changement de ton ou d'expression faciale

- Regarde l'adulte et établit un contact visuel

- Produit un son ou un geste pour attirer l'attention

- Regarde un objet/le montre pour l'obtenir

Utilise des moyens augmentatifs de communication en contexte de classe (pictogrammes, eye-tracking, Makaton, code gestuel)

\section{Manifestation du refus}

- Ne manifeste pas son approbation/son refus

- Manifeste son approbation/son refus par son comportement

- Sait dire " non " ou utilise un geste conventionnel

\section{Manifestation du plaisir/déplaisir}

\section{Aspects relationnels}

- Manifeste sa crainte envers des personnes inconnues

- Établit un lien privilégié avec un (ou des) professionnel(s) et/ou avec un(e) camarade

- Autre: Inhibition ou avidité relationnelle; Affinités électives manifestes; Excitation dans les contextes relationnels

La qualité des échanges émotionnels et corporels prend une place prépondérante dans la communication. Si l'intention de communiquer est présente chez la plupart des jeunes, elle se manifeste sous des formes parfois difficiles à décoder: des sons, des mimiques, des gestes ou des manifestations corporelles ou comportementales, discrètes ou au contraire très massives. Le contact visuel, en absence de cécité, constitue, pour plus de la moitié d'entre eux, une modalité de communication privilégiée, même si sept d'entre eux produisent un son ou un geste pour attirer l'attention, ou peuvent même désigner un objet pour l'obtenir. Des difficultés dans le processus de régulation tonico-émotionnelle sont rapportées chez une grande majorité des jeunes de l'étude, mais elles n'empêchent pas toute expérience et toute expression d'émotion fonctionnelle. 
Des possibilités de représentation sont également présentes chez tous les jeunes mais traduisent des niveaux d'organisation psychique extrêmement différents, allant de la représentation en actes moteurs à la représentation iconique ou à son émergence, jusqu'à la représentation symbolique pour seulement quatre d'entre eux. Les capacités d'extraction des invariants diffèrent aussi de manière importante: ainsi par exemple, on observe chez presque tous les jeunes (17 sur 18) des conduites d'orientation et d'attention en direction d'objets (matériels ou humains) qui témoignent d'un véritable intérêt pour l'environnement et les apprentissages, mais chez seulement la moitié d'entre eux des postures finalisées orientées vers un objet (spontanément ou en réponse à une sollicitation).

La construction des invariants, dont dépend la stabilité des représentations et leur développement constitue donc un point d'achoppement chez un grand nombre de ces jeunes. On peut faire l'hypothèse que les sensations restent alors brutes et morcelées, le jeune éprouvant de la difficulté à rassembler spontanément les qualités sensibles qui le renseignent sur son propre corps, sur les objets et les personnes de son environnement.

Ces résultats confirment à la fois l'existence de potentiels d'apprentissage effectifs, qui se révèlent au cours d'observations répétées et adaptées (Detraux, 2013) et la diversité des configurations cliniques. Le polyhandicap constitue de fait une réalité hétérogène à la fois aux plans intra et inter individuel, à laquelle les enseignants, les parents et les jeunes eux-mêmes doivent faire face. Par ailleurs, les troubles liés au polyhandicap sont intriqués et impactent à la fois le fonctionnement, le développement et l'ensemble des apprentissages. On peut ainsi avancer que la diversité des configurations individuelles va impliquer des stratégies de compensation et d'accompagnement du développement spécifiques, qui ne peuvent se réduire à la juxtaposition de réponses visant la réduction de chacun des troubles.

\section{l'étude longitudinale en contexte écologique de classe}

\section{Des processus d'apprentissage très dépendants du contexte pédagogique et relationnel}

Si, comme nous l'avons précédemment montré, des potentialités d'apprentissage existent bien chez tous ces jeunes, à des niveaux de développement extrêmement différents, qu'en est-il de leur actualisation, en contexte de classe?

Le tableau ci-dessous propose une synthèse de l'ensemble des comportements observés chez les élèves en contexte écologique de classe. Ils témoignent de manifestations très variées, d'un enfant à l'autre, mais également très fluctuantes en fonction du contexte (relationnel, pédagogique) et de la disponibilité psychique du jeune (facteurs individuels). 
Tableau 3: Observations en contexte écologique de classe

États de vigilance et disponibilité potentielle aux situations d'apprentissage

\begin{tabular}{|l|l|}
\hline $\begin{array}{l}\text { Comportements observés } \\
\text { (moteurs, cognitifs, émotionnels) }\end{array}$ & Exemples \\
\hline Endormi, somnolent & Garde les yeux fermés \\
\hline Éveillé (actif, passif) & Immobile, en mouvement \\
\hline Crises épilepsie ou absences & Myoclonies, absences \\
\hline Auto-stimulation, auto-agression & Se tape la tête \\
\hline Débordement émotionnel & Crie, s'agite \\
\hline
\end{tabular}

En réponse à une sollicitation (envers un adulte, un pair, un objet)

\begin{tabular}{|l|l|}
\hline $\begin{array}{l}\text { Comportements observés } \\
\text { (moteurs, cognitifs, émotionnels) }\end{array}$ & Exemples \\
\hline Expressions facials & Peur, colère \\
\hline Vocalisations, émissions sonores, mots & Chante, vocalise, parle \\
\hline Manifestation de Plaisir/déplaisir & Sourit, pleure \\
\hline Regard/absence de regard & Suit du regard, regarde \\
\hline Mouvements corporels & Se tourne, se détourne \\
\hline $\begin{array}{l}\text { Gestes orientés sur autrui, } \\
\text { ou sur un objet (intentionnalité) }\end{array}$ & Pointe, touche, explore \\
\hline $\begin{array}{l}\text { Succession d'actions motrices } \\
\text { organisées vers un but }\end{array}$ & Touche puis secoue l'objet \\
\hline Imitation & Reproduit le geste \\
\hline
\end{tabular}

À l'initiative de l'élève (envers un adulte, un pair, un objet)

\begin{tabular}{|l|l|}
\hline $\begin{array}{l}\text { Comportements observés } \\
\text { (moteurs, cognitifs, émotionnels) }\end{array}$ & Exemples \\
\hline Expressions facials & Peur, colère \\
\hline Vocalisations, mots & Chante, vocalise \\
\hline Manifestation de plaisir/déplaisir & Sourit, pleure \\
\hline Mouvements corporels & Se tourne, se détourne \\
\hline Gestes et actions motrices intentionnels & Prend, touche, explore \\
\hline $\begin{array}{l}\text { Succession de gestes, d'actions } \\
\text { motrices organisées vers un but }\end{array}$ & $\begin{array}{l}\text { Attrape l'objet, le secoue, le manipule } \\
\text { en l'absence de consigne donnée par } \\
\text { I'adulte }\end{array}$ \\
\hline Comportement d'imitation différée & $\begin{array}{l}\text { Reproduit une action de manière } \\
\text { spontanée }\end{array}$ \\
\hline
\end{tabular}


Une grande fluctuation des états d'éveil et une porosité importante à l'environnement

La fluctuation de certains états d'éveil (passage de l'état de somnolence à l'état d'éveil passif puis actif par exemple, ou encore passage de l'état d'auto-agression à l'état d'éveil actif) nous a permis de déceler la possibilité, pour certains jeunes, de tirer parti des étayages relationnels offerts par le contexte pour se réguler émotionnellement. Ainsi par exemple, Jules (7 ans) peut, à certains moments et grâce aux sollicitations de pair ou de l'enseignante, sortir de son état de somnolence pour se mobiliser plus activement sur la tâche proposée. Maxence ( 9 ans) et Inès (13,6 ans) réussissent à plusieurs reprises à se calmer lors de débordements émotionnels, grâce à l'étayage d'un professionnel qui leur parle doucement ou encore sous l'effet d'un contenant sonore (une chanson, une musique). Nous avons également pu analyser que la présence physique d'un professionnel aux côtés de l'apprenant pouvait se révéler contenante pour certains, permettant en particulier d'augmenter leur niveau de vigilance et d'attention ou, à l'inverse, surexcitante pour d'autres. Enfin, dans une même séance et pour un même élève, cette présence peut avoir des effets nocifs ou positifs, selon les moments, sans que l'on soit toujours en mesure d'interpréter de manière fiable les causes de ces changements.

Par ailleurs, nous avons observé chez six jeunes, des capacités à moduler leurs réactions tonico-émotionnelles en fonction de l'action du milieu externe: Clotilde (13 ans) et Driss (9 ans), par exemple, réagissent aux changements d'intonation, lorsqu'elle est félicitée après une réussite pour Clotilde, Iorsqu'on lui signifie fermement notre désaccord pour Driss. Driss peut également être sécurisé par la présence de l'enseignante, ce qui l'aide alors à limiter l'impact négatif des émotions dysfonctionnelles ressenties. La plupart des jeunes de l'étude montrent une très grande sensibilité (voire une hypersensibilité pour 9 d'entre eux) au contexte relationnel avec lequel ils entrent en résonnance, et nous avons pu observer des mouvements affectifs et émotionnels indiquant une grande porosité au climat émotionnel du groupe. Enfin nous avons pu analyser que les décharges tonico-émotionnelles (sursauts, brusque changement de posture, cris...) peuvent constituer une réponse à teneur émotionnelle évidente (peur, joie, surprise...) ou encore une modalité défensive (évitement, protection) alors que la crise tonico-émotionnelle qui se manifeste par un état de tension extrême (poings serrés, tremblements, mouvements paroxystiques) signe la défaillance complète du système de contenance et de pare-excitations. Ces résultats confirment l'importance du tiers régulateur externe pour étayer l'élève en situation de polyhandicap et l'aider dans ses difficultés de régulation émotionnelle, autant qu'ils incitent à la plus grande prudence concernant l'interprétation des comportements socio-émotionnels.

Une relation pédagogique qui assure des fonctions de médiation, d'étayage et de contenance active

À partir de l'analyse des comportements des jeunes en contexte écologique de classe, nous avons pu mettre en évidence que la mobilisation par les élèves polyhandicapés des processus sous-jacents aux apprentissages comme l'attention, la mémoire, les processus de représentation et de régulation socio-émotionnelle, dépend étroitement du contexte et de l'environnement humain interagissant avec 


\section{DOSSIER}

l'élève. Là où l'attention portée au développement et à l'autonomie du jeune suffit à soutenir une dynamique structurante d'interrelation, le contexte de polyhandicap nécessite de réfléchir aux conditions requises pour y parvenir. Parmi ces conditions, nous retenons:

- L'établissement de liens intersubjectifs favorisant une dynamique d'échanges fonctionnels. C'est pour nous le premier enjeu de l'accessibilité pédagogique. La qualité des échanges émotionnels et corporels prend une place prépondérante dans la communication, et les difficultés à interpréter les signes discrets de communication constituent un point d'achoppement au développement des apprentissages. Une bonne connaissance des modalités de communication (en émission et en réception) s'avère donc indispensable, de même qu'une bonne connaissance des styles personnels d'apprentissage. Pourtant, même si l'enseignant est conscient de la dimension subjective des élèves (beaucoup ont pu témoigner des affinités particulières à chacun, des sensibilités propres aux jeunes, etc.) la relation pédagogique est particulièrement déséquilibrée, par manque de feed-back (absence de parole, difficultés d'interprétation...) : I'adulte devra non seulement porter les intentions, énoncer les contenus, mais aussi créer les conditions de I'interrelation, maintenir les liens (sociaux ou de pensée), stabiliser les concepts, les perceptions, les sensations, les expériences, et relier entre elles ces différents contenus de pensées et d'action pour composer un environnement qui prenne sens. Sans ces opérations de l'adulte, l'enfant ne peut prendre des points de repère assez solides pour construire, selon son profil, son éprouvé entitaire ou son sentiment identitaire, ses proto-représentations ou ses représentations.

- Le deuxième enjeu de l'accessibilité pédagogique va porter sur l'accès cognitif à l'environnement scolaire, qu'il soit matériel et humain. Les enseignants cherchent à organiser les conditions d'un meilleur accès perceptif en tenant compte des particularités propres à chaque élève en situation de polyhandicap: ils vont s'assurer par exemple que les objets de l'environnement sont à la portée des sens de l'élève, de manière à ce que ceux-ci en perçoivent l'existence physique, et aient ainsi l'opportunité de s'en construire une représentation. Par ailleurs, étant donné les perturbations de la régulation tonico-émotionnelle qui entraînent des difficultés de régulation des émotions, et le défaut d'équipement cognitif, les élèves en situation de polyhandicap montrent des difficultés à discriminer, dégager des invariants, et mettre en place une capacité stable de représentation, spontanément. Dans un tel contexte, on comprend aisément l'importance et la spécificité de la médiation humaine: pour aider l'enfant à ignorer certaines stimulations, à en détecter d'autres, à mettre de l'ordre dans l'abondance perçue, à distinguer les événements interdépendants de ceux qui n'existent que pour eux-mêmes, à construire du sens à partir de la complexité ambiante sans se laisser encombrer ou déborder par elle. L'analyse de l'activité des professionnels nous a permis de constater l'importance accordée au moment et à la manière d'introduire les objets, de façon à les rendre suffisamment saillants pour être d'abord perçus puis explorer de différentes manières par l'élève. La prise en compte de la disponibilité du jeune constitue dans ce contexte un indice de sa mobilisation potentielle aux apprentissages. 
- Un travail de contenance active. L'instauration de rituels, la présence des mêmes personnes, le lieu aménagé pour la classe, constituent autant de repères externes qui pallient la discontinuité des ressentis. De même, l'inscription de l'élève dans une temporalité (la répétition, le rappel, la projection vers un futur proche...) contribue à situer l'activité pédagogique au-delà de la circularité de la relation au corps propre. À la suite de Mellier (2005), nous définirons la contenance comme un véritable travail psychique de transformation que les parents et les professionnels ont à réaliser face aux souffrances très primitives d'un enfant polyhandicapé. L'observation constitue ici un acte professionnel majeur. Le décodage des expressions du visage entre l'adulte (le professionnel) et l'élève aboutit à classer les actes de l'enfant polyhandicapé comme communicatifs parce que le professionnel est attentif et répond à ces actes comme s'ils étaient intentionnels et significatifs. De ce fait, ils s'inscrivent progressivement dans les codes répertoriés et intuitivement reconnus. Le décodage est une façon de communiquer de façon stable les signaux chez soi et chez les autres. Ainsi, la communication garde tout son sens en la situant au cœur de la contenance, donc proche de la compréhension.

\section{Des processus d'apprentissage qui témoignent de profils psychodéveloppementaux et de styles personnels d'apprentissage très différents}

L'observation minutieuse et continue des 18 jeunes de notre étude, en contexte écologique de classe, nous a permis de repérer des processus d'apprentissage, et certaines évolutions dans les comportements observés. Nous constatons, avec leur hétérogénéité, que les processus d'apprentissage témoignent de profils psychodéveloppementaux très divers. À titre d'exemple, nous présentons ici une synthèse des observations concernant trois jeunes dont le profil psychodéveloppemental de polyhandicap est proche, même si leurs comportements témoignent de manières d'être au monde singulières:

Pour ces trois jeunes, il reste très difficile d'interpréter de manière fiable leurs expressions et comportements. On observe peu d'actions motrices organisées vers un but, les moments de présence au monde externe sont ténus et encore difficilement évaluables. Certaines évolutions ont été cependant repérées: davantage de signes de disponibilité potentielle, une curiosité et un intérêt croissant pour l'environnement de la classe. Par exemple même si l'intérêt pour autrui n'est toujours pas décelable chez Jules en fin de recherche, celui-ci a développé au fil de l'année des compétences tactiles exploratoires. II commence à s'intéresser au ballon multisensoriel de la classe quand il est présenté devant lui, et qu'on l'y encourage en orientant ses mains. On observe également des signes discrets de mémorisation, il a répété chez lui des gestes appris en classe, de manière spontanée. Janine est devenue pour sa part plus sensible à la présence de certains adultes de la classe, dont l'étayage lui permet de soutenir son exploration naissante. Enfin Max commence à manifester de l'intérêt pour ses pairs lors des temps de classe. Au niveau socio-émotionnel, des capacités d'expression émergent chez Janine (elle peut maintenant pleurer lorsqu'elle est douloureuse). Cette ouverture au monde se traduit chez ces jeunes par des mouvements corporels qui commencent à s'organiser. On peut faire l'hypothèse que la répétition des rituels en classe a soutenu leurs capacités d'attention et leur ouverture à l'environnement, notamment à leurs pairs. 


\section{DOSSIER}

Nous avons pu globalement constater que les enjeux d'apprentissage, pourtant propres à chacun des jeunes, rejoignent des préoccupations liées aux grand domaines de I'apprentissage et concernant ce que Vygotski a pu définir comme la zone de proche développement. Cet espace, défini par l'écart entre les compétences actuelles et celles qui peuvent être atteintes avec l'aide d'un adulte médiateur, est celui dans lequel s'incarne l'activité pédagogique.

Les enjeux de l'apprentissage se situent également, pour l'enseignant, dans la connaissance qu'il va pouvoir acquérir (ou non) de la personnalité de ses élèves, en particulier de leur style personnel d'apprentissage, de leurs préférences ou aversions, de leurs appétences spécifiques ou difficultés propres. Les styles d'apprentissage sont caractérisés par les modes d'entrée sensoriels (auditifs, visuels...), leur mantèlement - déficitaire ou non -, et les modes relationnels dans les apprentissages (appétence ou rejet de la relation). Ils entreraient en concordance avec des propositions caractérisées par les enjeux propres à leur niveau de développement (enactif, iconique ou symbolique). Ainsi la question des rythmes circadiens ne concerne que le jeune Jules (profil I). Le plaisir à agir est mentionné comme un élément potentiellement moteur pour Nolan, de même que la nouveauté pour Clotilde (profil II). D'autres enjeux au contraire sont partagés par un plus grand nombre de jeunes, soit à l'intérieur même des profils, soit entre les différents profils. Les rencontres, programmées ou issues d'opportunités provoquées dans la régularité des situations d'apprentissage, entre styles d'apprentissage et enjeux (donc liés aux profils développementaux), permettraient à petits pas la poursuite de processus de développement, desserrant ainsi l'étau de la situation de polyhandicap.

\section{CONCLUSION}

Partant d'observations particulières sur un échantillon réduit, notre étude comporte un certain nombre de limites, malgré les précautions méthodologiques prises (double codage, lectures multiples des données brutes, analyse en regards croisés). Parmi ces limites retenons principalement la difficulté à interpréter des signes parfois très discrets, et à accéder à la subjectivité de l'élève en situation de polyhandicap. Nous avons pu repérer que le polyhandicap rend fragiles les capacités identificatoires du professionnel envers le jeune, ainsi que les capacités expressives de l'enfant ou de l'adolescent envers lui. Parmi les enjeux de l'apprentissage, les difficultés de communication apparaissent prépondérantes, et nécessiteraient des études plus approfondies, en contexte scolaire.

Rejoignant les travaux de Scelles (2013), nous pensons qu'une démarche pédagogique doit d'abord s'étayer sur la prise en compte des déficits sensoriels et moteurs liés au polyhandicap, mais aussi sur les particularités de la communication. L'évaluation globale des potentialités du jeune constitue donc une première étape cruciale, qui permet d'envisager des objectifs individuels d'apprentissage à partir d'une analyse fonctionnelle des besoins développementaux. Notre étude minutieuse des processus sous-jacents aux apprentissages nous invite à accorder une place importante aux émotions et à l'expérience sensorielle dans l'évaluation de la cognition. Nous avons pu montrer par exemple que la mémoire et l'attention sont fortement influencés par la 
résonance émotionnelle de la personne polyhandicapée à l'action de l'environnement, et par ailleurs toujours difficiles à stabiliser. L'environnement humain peut ainsi entraver ces processus d'apprentissage ou au contraire contribuer à les développer grâce à des stratégies pour compenser certaines déficiences, maintenir un niveau suffisant de motivation chez le jeune, le sécuriser suffisamment, voire, dans certaines situations, contribuer à abaisser son niveau de tensions. Notre étude nous conduit par ailleurs à privilégier une perspective dynamique dans la manière de concevoir l'enseignement. Nous rejoignons ici les analyses produites par de nombreux chercheurs qui engagent à s'intéresser à la manière dont la personne polyhandicapée construit ses connaissances, profite des aides, sélectionne et mobilise des stratégies pour résoudre les problèmes ou agir sur l'environnement, et pas seulement à l'évaluation de ses compétences et de ses performances (Petitpierre et Scelles, 2013). Enfin, nous avons constaté que la scolarisation sans pré-requis exigés, préconisée par un certain nombre de professionnels, n'est pas remise en question par les résultats de l'étude: aucun jeune n'a régressé à l'issue de l'expérience de scolarisation, et la plupart témoignent de changements favorables à leur développement dans les activités de connaissance de soi, des autres et des objets culturels.

\section{Références}

Auvray, M. (2005). La suppléance perceptive. Implications sur les définitions de nos modalités sensorielles. Voir, 30-31, 2-13.

Bullinger, A. (2007). Le développement sensori-moteur de l'enfant et ses avatars: Un parcours de recherche. Toulouse: Érès.

Blondel, F., et Delzescaux, S. (2018). Aux confins de la grande dépendance. Le polyhandicap, entre reconnaissance et déni d'altérité. Toulouse: Érès.

Chard, M., et Roulin, J. (2015). Vers une meilleure compréhension du fonctionnement cognitif des personnes polyhandicapées. Revue francophone de la déficience intellectuelle, 26, 29-44.

Bruner, J. S. (1968). Processes of cognitive growth: Infancy. Clark University Press.

Dind, J. (2018). Les manifestations de la conscience de soi chez les enfants polyhandicapés (Thèse de doctorat non publiée). Université de Fribourg, Suisse.

Detraux, J.-J. (2013). L'approche évaluative comme base de l'intervention éducative auprès de personnes polyhandicapées. In R. Scelles et G. Petitpierre, Polyhandicap: processus d'évaluation cognitive (pp.101-120). Paris: Dunod.

Engel, G.-L. (1977). The need for a new medical model: a challenge for biomedicine. Science, 196. Issue 4286, 129-136.

Hostyn I., \& Maes, B. (2009). Interaction between persons with profound intellectual and multiple disabilities and their partners: A literature review. Journal of Intellectual and Developmental Disability, 34, 366-376.

Hostyn I., Neerinkx, H., \& Maes, B. (2011). Attentional processes in interactions between people with profound intellectual and multiple disabilities and direct support staff. Reseach of Developmental Disability, 32(2), 491-503.

Korff-Sausse, S. (2008). Responsabilité éthique du point de vue de la personne handicapée elle-même. In R. Scelles et al., Handicap: I'éthique dans les pratiques cliniques (pp.77-86). Toulouse: Érès. 


\section{DOSSIER}

Mellier, D. (2005). La fonction à contenir: Objet, processus, dispositif et cadre institutionnel. La psychiatrie de l'enfant, 48(2), 425-499.

Neerinckx, H., \& Maes, B. (2016). Joint attention behaviours in people with profound intellectual and multiple disabilities: the influence of the context. Journal of Applied Research in Intellectual Disabilities, 29(6), 574-584.

Nijs, S., Vlaskamp, C., \& Maes, B. (2015). Children with PIMD in interaction with peers with PIMD or siblings. Journal of Intellectual Disability Research, 60(1), 28-42.

Ouss, L. (2019). Quelle psychopathologie des handicaps neurologiques précoces? In A. Boissel, Quand le handicap s'invite au cours de la vie (pp. 79-96). Toulouse: Érès.

Pereira Da Costa, M., et Scelles, R. (2012). Un outil d'évaluation des compétences cognitives des jeunes polyhandicapés: le P2CJP. Alter, Revue européenne de recherche sur le handicap, 6(2), 110-123.

Petitpierre-Jost, G. (2005). Programmes de stimulation pour personnes polyhandicapées: suggestions pour l'amélioration du cadre d'application pédagogique et thérapeutique. Devenir, 17(1), 39-53.

Petitpierre, G. (2011). De la manière de concevoir le développement à la mise en place de situations d'apprentissage cognitif. La nouvelle revue de l'adaptation et de la scolarisation, Hors-série $n^{\circ} 6,147-158$.

Ponsot, G., et Boutin, A.-M. (2017). Le polyhandicap, une situation particulière de handicap. In P. Camberlein et G. Ponsot, La personne polyhandicapée. La connaître, I'accompagner, la soigner (pp. 225-247). Paris: Dunod.

Toubert-Duffort, D. (2011). Appareillages institutionnels et polyhandicap. La nouvelle revue de l'adaptation et de la scolarisation, hors-série $n^{\circ} 6,97-107$.

Toubert-Duffort, D. (2017). Les principes et les modalités de la scolarisation des enfants polyhandicapés. In P. Camberlein et G. Ponsot, La personne polyhandicapée. La connaître, l'accompagner, la soigner (pp. 375-384). Dunod.

Saulus, G. (2007). La clinique du polyhandicap comme paradigme des cliniques de l'extrême. Champ psy, 45(1), 125-139.

Saulus, G. (2008). Modèle structural du polyhandicap, ou : comment le polyhandicap vient-il aux enfants? La psychiatrie de l'enfant, 51, 153-191.

Saulus, G. (2009). Le concept d'éprouvé d'existence. Contribution à une meilleure lecture des particularités psychodéveloppementales du polyhandicap. In S. Korff-Sausse (dir.), La vie psychique des personnes handicapées (pp. 25-44). Toulouse: Érès.

Saulus, G. (2017). L'activité de conscience chez les personnes polyhandicapées. In P. Camberlein et G. Ponsot, La personne polyhandicapée. La connaître, I'accompagner, la soigner (pp. 225-247). Paris: Dunod.

Suchman, L. (1987). Plans and situated action. Cambridge, MA: Cambridge University Press.

Vygotski, L. (1997). Pensée et langage (Sève F., trad.). Paris: La Dispute. (CEuvre originale publiée en 1934).

Vurpillot, E. (1972). Les perceptions du nourrisson. Paris: PUF.

Weil-Barais, A., et Resta-Schweitzer, M. (2008). Approche cognitive et développementale de la médiation en contexte d'enseignement-apprentissage. La nouvelle revue de l'adaptation et de la scolarisation, 42(2), 83-98. 